\title{
Innovación social: Factores claves para su desarrollo en los territorios
}

\author{
Víctor García-Flores \\ Luis Palma Martos
}

RESUMEN: Existe un amplio consenso sobre que la innovación social es un fenómeno necesario para contribuir a resolver los nuevos retos sociales de carácter complejo y multidimensional que han proliferado en las últimas décadas. Sin embargo, y aunque la innovación social ha ido ganando peso, no hay acuerdo general sobre qué es, ni se conocen en profundidad todos los factores que determinan su aparición en un territorio.

En el contexto descrito, este trabajo tiene como objetivos principales hacer una revisión del estado del arte, proponer una definición de innovación social e identificar y analizar la importancia de aquellos factores que favorecen su nacimiento y desarrollo en el territorio. Con este fin, se ha utilizado una metodología cualitativa a través de la revisión sistemática de la literatura y la realización de 24 entrevistas semiestructuradas a agentes inmersos en procesos de innovación social. Las entrevistas, una vez transcritas, fueron analizadas con el programa Atlas.ti, lo que ha permitido proponer una definición de innovación social y plantear cinco grandes pilares (elementos sociales y culturales; apoyo político e institucional; conocimiento y mecanismos facilitadores; componentes espaciales y entidades; y mecanismos que determinan la estructura productiva empresarial y social), que agrupan 29 factores identificados y ponderados en función del número de veces que fueron señalados por los entrevistados. Finalmente, se ha realizado un análisis interpretativo de los resultados en el que se explica la relevancia de cada uno de los factores y la relación existente entre ellos.

PALABRAS CLAVE: Innovación social, tercer sector, sector público, análisis cualitativo, economía social, territorio.

CLAVES ECONLIT: A13, 030, P35, P36.

Cómo citar este artículo / How to cite this article: GARCÍA-FLORES, V. \& PALMA, L. (2019): "Innovación social:

Factores claves para su desarrollo en los territorios", CIRIEC-España, Revista de Economía Pública, Social y Cooperativa, 97, 245-278. DOI: 10.7203/CIRIEC-E.97.14148.

Correspondencia: Víctor García-Flores, e-mail: vgarcia10@us.es (contacto), y Luis Palma Martos, Ipalma@us.es,

Universidad de Sevilla. 


\section{EXPANDED ABSTRACT}

\section{Social innovation: Key factors for its development in the territories}

Currently, the term innovation is often associated with the generation of new technologies, excluding other forms of innovation, with equal or greater value, such as social innovation.

Technological innovation has been repeatedly used as a means differentiation in order to achieve a higher enterprise competitiveness. This has led to achievement of great scientific and technical progress. Nevertheless, in the last few decades, this has not prevented some territories from social issues such as poverty, exclusion, inequality, deterioration of environmental conditions and unemployment, among others. All these problems have a high local impact, being here where social innovation importance as a force for potential change is growing.

Despite the broad consensus regarding the potential and the positive effects that social innovation has in those territories, there is still considerable debate surrounding how this concept should be defined and what factors contribute to its appearance. There are many variables that have been mentioned in the literature; however, a review of previous works shows that it is an open field for the identification of new ones.

As described above, this paper has three main aims: to analyze the state of the art up to now, to give a definition of social innovation and to identify, analyze and weigh those key factors for its emergence in the territories.

To this end we have designed a methodology based on a series of qualitative analysis techniques. Firstly, a review of the state of the art has been carried out to obtain a first approximation to the concept of social innovation and determine which elements are decisive in its appearance. Secondly, based on the existing literature and knowing what the problem is, we complete the methodology by designing and conducting semi-structured interviews. We chose this technique because we understand that social innovation is a living and constantly evolving phenomenon. This makes it very useful and allows us to confront the ideas and evidence from our literature review in a pragmatic way. The information gathered from the interviews allow us to identify, in a reliable and empirical way, new key elements to define social innovation. Additionally, the interviews help us to recognize and weigh the existence of new essential factors for the development of social innovation.

These interviews have been conducted with a total of 24 agents involved in social innovation processes: managers and directors of centers responsible for promoting social innovation; agents that developed socially innovative initiatives; and scientists and academics who published work in this field. 
To analyze the content of the 24 interviews, we used the Atlas.ti computer program. This tool helped us analyze the transcripts. In order to explore and interpret the texts we analyzed 24 primary archives, which represent one transcript for each interview carried out. Afterwards, we encoded them. This process is a form of data reduction, since, based on a large volume of information, we managed to segment certain pieces of information (quotes) and label them based on codes. These are the main elements of analysis and they are considered as a system of categories. In our case, each time we have identified a quote, which made reference to the existence of a factor that conditioned the irruption of social innovation or that enunciated characteristics that defined it, we labeled a code -referred to a certain factor related to the content of the quotes-.

Once the individual coding and data editing was completed, we organized the codes into families. Specifically, in line with the main purposes of our analysis, in two families: one to collect the codes related to the definition of social innovation and a second to gather those related to its determinant factors.

To provide our study with greater depth, it was essential to carry out a statistical analysis of the content that would allow us to know the frequency of appearance of the codes. First, we calculated the absolute frequency of the codes, referred to the number of times a quote was identified with the same code. Nevertheless, to obtain more information about the importance of each one of the codes, we relativized them considering that they had previously been divided into families. Thus, we have obtained the relative frequency by families, which is, the number of times any code is repeated within a family according to the total number of times codes included in that family appear. This contributes directly to know which factors have more strength in the emergence of social innovation, among the ones pointed out by the interviewers.

After the performance of the aforementioned analysis, we suggest the following definition of social innovation: Practices or initiatives carried out by the community that, based on the products, services or models they generate, or through the process designed to achieve their objectives, they reach solutions that give a better response, in an alternative and creative way, to social problems or needs. In addition, we point out five key points for the development of social innovation: social and cultural elements; political and institutional support; knowledge and facilitating mechanisms; spatial components and entities; and mechanisms that determine the corporate and social productive structure. These gather 29 factors identified and weighted according to the number of times they were indicated by the interviewees.

Therefore, this work, not only points out the key factors of social innovation and the foundations on which they are grouped by theme, but also shows the importance of each underlying determinant according to its absolute and relative frequencies. These frequencies are used as a proxy to weigh the importance of these factors and foundations. This allows to know which ones are more needed and have more weight in the emergence of social innovations. 
The ten factors with more impact are: the socioeconomic level of the population (0.09532), the existence of strategies and lines of action to develop social innovation (0.08838), the existence of laboratories or centers of social innovation (0.05892), the existence of public funding (0.05892), the existence of social concern $(0.05719)$, the cultural propensity to change $(0.05719)$, knowledge of the local reality $(0.05545)$, knowledge of the opportunities and potential of new means and instruments $(0.04506)$, links encouraging public-private relations $(0.04332)$ and the existence of cooperatives $(0.03986)$.

In summary, it should be noted that the recognition of these factors, together with the weighing of their relative importance, represents a considerable innovation with respect to the existing texts. This work has allowed us to verify the suitability of some determining factors that had previously been pointed out in other studies, state new ones that emerged after the investigation, explain the correlation between them and present how important and what role each of them plays to have a beneficial ecosystem for social innovation. This knowledge can be very useful when establishing lines and criteria for the design of prioritization strategies, useful where there are restrictions of a political, organizational or budgetary nature.

KEYWORDS: Social innovation, third sector, public sector, qualitative analysis, social economy, territorory. 


\section{Introducción}

En la actualidad, en numerosas ocasiones, el término innovación se asocia directamente con la generación de nuevas tecnologías, dejando de lado otros tipos de innovación, de igual o mayor envergadura, como puede ser la innovación social.

La innovación tecnológica ha sido utilizada recurrentemente como medio de diferenciación para lograr una mayor competitividad empresarial, lo que ha conllevado la consecución de grandes avances científico-técnicos. Sin embargo, esto no ha evitado que en las últimas décadas emerjan grandes retos sociales en los territorios: pobreza, exclusión, desigualdad, deterioro de las condiciones medioambientales y desempleo, entre otros. Todos ellos son problemas que tienen una alta repercusión en el ámbito local, siendo éste el contexto donde la innovación social gana más peso como fenómeno con potencial transformador.

Por ello, entendemos que centrar los sistemas de innovación ${ }^{1}$ únicamente en conseguir mejoras tecnológicas, aunque productivo y eficaz económicamente, no es del todo eficiente, debido a la falta de respuesta existente ante los retos y problemas sociales de carácter complejo y multidimensional. Esto justifica por qué la innovación social, que se presenta como una solución participativa y cooperativa, es un fenómeno que debe ser fomentado para coadyuvar a resolver los retos actuales que amenazan el bienestar y la misma estabilidad de la sociedad.

Existe un amplio consenso acerca de las potencialidades y los efectos positivos que genera la innovación social en los territorios. En cambio, la falta de acuerdo es latente cuando se trata de definirla y conocer qué factores contribuyen a que aparezca. Son muchas las variables que han sido mencionadas en diferentes trabajos, pero, en cambio, la revisión de la literatura evidencia que es un campo abierto a la identificación de otras nuevas.

Este trabajo nace desde el convencimiento de la necesidad de averiguar los factores más significativos que son necesarios para crear un ecosistema que facilite la aparición de innovaciones sociales. Por esta razón, nosotros vamos más allá de enunciar lo que sería una definición de síntesis y de limitarnos a reproducir factores que previamente ya han sido señalados. En este contexto, son tres los objetivos principales: realizar un análisis del estado del arte hasta la actualidad, proponer una definición de innovación social e identificar y analizar, según su importancia, factores claves para la irrupción de la innovación social en los territorios. 
Con este fin hemos diseñado una metodología con entrevistas semiestructuradas realizadas a un total de 24 agentes involucrados en procesos de innovación social: responsables y directores de centros encargados en promover la innovación social; agentes que han desarrollado iniciativas socialmente innovadoras; y científicos y académicos que han publicado trabajos de impacto sobre la materia. Desde nuestro punto de vista, consideramos que tener en cuenta la visión de aquellos que están en primera línea, trabajando día a día con la innovación social, es la forma más adecuada para conocer la realidad de la manera más próxima y fidedigna.

Entre las principales contribuciones de este trabajo se encuentran: i) proponer una nueva definición de innovación social, enunciando tres postulados esenciales que debe tener cualquier práctica socialmente innovadora, ii) comprobar la idoneidad de ciertos factores que ya habían sido señalados en la literatura como determinantes de la innovación social, iii) detectar nuevos factores que no habían sido identificados hasta ahora, iv) ponderar todos ellos para conocer la relevancia de cada uno en la aparición de la innovación social y v) explicar por qué facilitan el desarrollo de las mencionadas prácticas socialmente innovadoras.

Finalmente, nos gustaría aclarar la estructura que hemos planteado para la realización de este trabajo: un segundo epígrafe, posterior a esta introducción, en el que se realiza una aproximación contextual; un tercer epígrafe, en el que se hace una revisión del estado de la cuestión, prestando especial atención a los diferentes puntos de vista desde los que la innovación social puede ser entendida, al elemento territorial y a los principales factores señalados como determinantes de ésta; un cuarto epígrafe en el que se presenta la metodología usada para la elaboración del presente artículo; un quinto epígrafe en el que se muestran y discuten los resultados obtenidos; y, por último, se exponen las principales conclusiones y la bibliografía utilizada.

\section{La emergencia de la Innovación Social. Una aproximación contextual}

La comprensión de las actividades de innovación y de su impacto socioeconómico ha sido objeto de estudio en numerosas investigaciones a lo largo de las últimas décadas. De hecho, existe una creciente literatura que trata la cuestión, y que, con todo, no lo ha logrado hacer de una forma totalmente eficiente e integradora.

Las deficiencias a la hora de abordar el concepto de innovación se derivan, principalmente, de la existencia de muy diversos tipos de ésta. Por ejemplo, la Organización para la Cooperación y el Desarrollo Económico (OCDE, de ahora en adelante) elaboró por primera vez en 1992 el Manual de Oslo, donde sólo se recogían como actividades de innovación las denominadas innovaciones tecno- 
lógicas y de procesos. En la segunda publicación de dicho Manual, en 1995, se incluyó la innovación en el sector servicios, y ya en la tercera edición de 2005 se realiza una redefinición de conceptos y se consideran la innovación en mercadotecnia y la innovación organizativa (Echeverría, 2008). Aunque mucho más completa, esta última sigue sin abordar la innovación de forma integral, haciéndolo desde un punto de vista muy empresarial, a pesar de que, en el propio documento la OCDE (2005: 23) reconoce que "la innovación es también importante para el sector público, aunque se sabe menos sobre estos procesos en los sectores no orientados al mercado". Se evidencia así que la OCDE conoce la existencia de más tipos de innovación, pero que hay un vacío de conocimiento sobre las funcionalidades y los efectos positivos que pueden ser conseguidos con otros tipos de innovaciones, como son las sociales.

No obstante, aun cuando los aspectos sociales de la innovación hayan pasado en muchas ocasiones desapercibidos, debido a que los enfoques más clásicos los han dejado sistemáticamente olvidados, las innovaciones sociales se han ido desarrollando a lo largo de la historia cuando se han pretendido resolver retos sociales (López y González, 2013). De hecho, Zubero (2015:14) subraya que "la innovación social es tan característica de las sociedades humanas vivas que sería un error reducirla a una práctica exclusiva de las denominadas sociedades modernas".

Muestra de la existencia de este proceso histórico es la creación en el siglo XIX de los microcréditos, de los sindicatos obreros o de los nuevos modelos de producción y distribución basados en el cooperativismo. También durante las primeras décadas del siglo XX destacan la aparición de sistemas de ayudas sociales o de atención infantil. Sin embargo, este fenómeno no ha comenzado a atraer la atención institucional hasta finales de la década de los 80 del siglo pasado. Este hecho es primordial para el desarrollo y fomento de las innovaciones, ya que como señalan Yépez (2017:45) "la influencia del aspecto institucional debe ser tenida en cuenta cuando se reflexiona acerca de la capacidad innovadora de los países y las regiones en el marco teórico de los sistemas de innovación".

En este sentido, destacan como los primeros centros e institutos con objeto de promover la innovación social, el Centre de recherche sur les innovations sociales (CRISES), que fue puesto en marcha en Canadá en 1986, y el Vienna-based Zentrum fur soziale Innovation (SZI) inaugurado en Austria en 1990 (Paz, 2015). A partir de entonces el paradigma schumpeteriano de innovación predominante, que entendía la sociedad como el ámbito donde se difundían las innovaciones, pero no contemplaba que pudiera tener el papel de agente innovador, se comienza a superar. Ciertamente, la mutación del mencionado paradigma empieza a consolidarse a finales del siglo XX, cuando se produce, según apuntan Echeverría y Merino (2011), un "giro social en los estudios de innovación"y en países como Australia, Nueva Zelanda, Canadá y Gran Bretaña comienza a defenderse la innovación social como alternativa y complemento a la innovación tecnológica.

En este proceso, en el que las instituciones comienzan a prestar atención a la innovación social, la OCDE en el año 2000 creó un Foro de Innovación Social dentro del Programa Local Economic and Employment Development (LEED), en el cual participaban 11 organizaciones de 6 países miem- 
bros, con el objetivo principal de facilitar la difusión internacional y la transferencia de las mejores políticas y prácticas en innovación social.

En esta línea, en la región de Quebec (Canadá) en 1997, el gobierno encargó la realización de un estudio sobre las posibles contribuciones de las ciencias sociales y las humanidades a una política planificada de innovación (Paz, 2015). A este respecto, las potencialidades de la innovación social para lograr un mayor dinamismo y desarrollo territorial han sido indicadas desde principios del siglo XXI por Méndez (2002), Hillier et al (2004) y Moulaert et al (2005).

Otro momento significativo es la publicación del estudio de Mulgan (2006), ya que se distingue claramente entre innovación social y empresarial. Apuntan Echeverría y Merino (2011: 1034) que este trabajo elaborado para la Young Foundation fue un apoyo teórico importante para la estrategia política del primer ministro Tony Blair cuando creo la Cabinet Office of the Third Sector.

En 2009 se puso a funcionar la Office of Social Innovation por parte de la administración de los Estados Unidos, cuyo fin principal era centrarse en el desarrollo de políticas y programas para acelerar la recuperación económica y fortalecer las comunidades.

Estos hitos fueron embrionarios para que, en 2010, la Comisión Europea, cuando da luz a la Innovation Union Initiative, incluya la innovación social como un objetivo a tener en consideración para abordar todo un conjunto de problemas y retos socioeconómicos que estaban desafiando la vida en los territorios. La emergencia de este fenómeno se consolida con la publicación en 2013 del Social Innovation Package y la Guide to Social Innovation. Dichas acciones han facilitado la transformación de la visión de la innovación en las regiones de Europa y han contribuido a la familiarización, desarrollo y aceptación de la innovación social para el logro de las metas marcadas en la Estrategia Europa 2020.

Todos estos hechos han provocado que, actualmente, la innovación social sea una prioridad para muchos gobernantes, y se encuentren centros para fomentarla en países como Canadá, Reino Unido, Austria, Estados Unidos, Alemania, Sudáfrica, Colombia, Chile y España, entre otros. 


\section{Revisión de la literatura}

\subsection{Innovación social}

A pesar de la profusión de los estudios acerca de qué es la innovación social, actualmente, hay una evidente falta de consenso en la literatura acerca de su significado. Esto impide encuadrar el término en una definición concreta y generalmente aceptada (Mulgan, 2006; Zubero, 2015; Mosselaer, 2016; Hernández-Ascanio et al, 2016; Palma et al, 2017; Salom-Carrasco et al, 2017). Tal es la falta de coincidencia existente acerca de cómo definirla, que Djellal y Gallouj (2012), llegaron a titular el primer epígrafe de su artículo Social Innovation and Service Innovation, como: "Social Innovation: the desperate quest for a definition".

Por esta razón, la consecución de una definición generalmente reconocida se ha convertido en la gran cuestión a abordar. Son muchos los autores que han definido la innovación social con análisis ciertamente solventes y de gran valor para el avance científico en la materia. No obstante, y dada la heterogeneidad de definiciones presentes en la literatura, nos parece fundamental contribuir desde nuestro trabajo a la búsqueda de nuevos elementos que permitan avanzar en discernir qué es la innovación social. Por ello, creemos necesario, tal y como se hace a partir de la tabla 1, hacer una revisión del estado de la cuestión. En ésta se recogen algunas de las definiciones más transcendentes de organismos nacionales e internacionales, así como de autores de referencia en la materia.

\section{Tabla 1. Distintas definiciones del concepto innovación social}

\begin{tabular}{|l|l|}
\hline Autor & Definición de innovación social \\
\hline Mulgan (2006:146) & $\begin{array}{l}\text { Son actividades y servicios innovadores que son llevados a cabo fundamentalmente por organizaciones } \\
\text { cuyo objetivo principal es social, y que tienen como fin satisfacer una necesidad o reto social. }\end{array}$ \\
\hline NESTA (2008) & $\begin{array}{l}\text { La innovación social consiste en desarrollar nuevas ideas para abordar problemas o necesidades sociales. } \\
\text { Puede ser un nuevo producto, servicio, iniciativa, modelo organizativo o un nuevo enfoque para la } \\
\text { prestación de servicios públicos. }\end{array}$ \\
\hline Pot y Vaas (2008: 468) & $\begin{array}{l}\text { Es un concepto más amplio que la innovación organizacional. Incluye cosas tales como la gestión } \\
\text { dinámica, la organización flexible, el trabajo más inteligente, el desarrollo de habilidades y competencias y } \\
\text { la creación de redes entre organizaciones. Es un complemento de la innovación tecnológica. }\end{array}$ \\
\hline Phills et al (2008:39) & $\begin{array}{l}\text { Una innovación social puede ser un producto, proceso de producción o tecnología (muy similar a la innova- } \\
\text { ción en general), pero también puede ser un principio, una idea, una legislación, un movimiento social, una } \\
\text { intervención o una combinación de ellos. Hace referencia a la innovación social como herramienta para } \\
\text { solucionar problemas colectivos, no individuales. }\end{array}$ \\
\hline
\end{tabular}




\begin{tabular}{|c|c|}
\hline $\begin{array}{l}\text { Sanchis y Campos } \\
(2008: 187)\end{array}$ & $\begin{array}{l}\text { Tiene por objeto la introducción de cambios relacionados con nuevas formas organizativas y de gestión } \\
\text { dentro de la organización. Entre sus principales objetivos figuran la inserción socio-laboral de personas, } \\
\text { principalmente con mayor riesgo de exclusión, y la protección y el respeto al medioambiente mediante el } \\
\text { crecimiento sostenible. }\end{array}$ \\
\hline $\begin{array}{l}\text { Harris y Albury } \\
(2009: 16)\end{array}$ & $\begin{array}{l}\text { Es una innovación beneficiosa, tanto para el bien social como para el interés público, siendo una innovación } \\
\text { inspirada en el deseo de satisfacer las necesidades sociales que pueden estar descuidadas por las formas } \\
\text { tradicionales de provisión del mercado, y que a menudo han sido mal atendidas o no resueltas por los } \\
\text { servicios organizados por el sector público. }\end{array}$ \\
\hline $\begin{array}{l}\text { Howaldt, y Schwarz } \\
(2010: 16)\end{array}$ & $\begin{array}{l}\text { Es una nueva combinación y/o nueva configuración de prácticas sociales en ciertas áreas de acción o } \\
\text { contextos sociales, impulsadas por ciertos actores o conjunto de ellos, de una manera intencionalmente } \\
\text { dirigida, con el objetivo de satisfacer mejor las necesidades y problemas sociales. }\end{array}$ \\
\hline Hubert (2010:33) & $\begin{array}{l}\text { Son aquellas nuevas ideas (productos, servicios y modelos) que simultáneamente satisfacen necesidades } \\
\text { sociales (de forma más efectiva que las alternativas), creando nuevas relaciones o colaboraciones. }\end{array}$ \\
\hline OCDE (2011:13) & $\begin{array}{l}\text { Son nuevos mecanismos y normas que consolidan y mejoran el bienestar de las personas, las comunidades } \\
\text { y los territorios, en términos de inclusión social, creación de empleo y calidad de vida. }\end{array}$ \\
\hline $\begin{array}{l}\text { Caulier-Grice et al } \\
(2012: 42)^{2}\end{array}$ & $\begin{array}{l}\text { Son las nuevas soluciones (productos, servicios, modelos, mercados y procesos, entre otros) que satisfacen } \\
\text { simultáneamente una necesidad social (de manera más efectiva que las soluciones existentes) y conducen } \\
\text { hacia nuevas o mejoradas relaciones y capacidades, así como a un mejor uso de los activos y recursos. }\end{array}$ \\
\hline $\begin{array}{l}\text { Oosterlynck et al } \\
(2013: 2)\end{array}$ & $\begin{array}{l}\text { Aquellas prácticas que suceden en ámbitos locales, que ayudan a personas en exclusión social y a grupos } \\
\text { sociales empobrecidos, a satisfacer necesidades sociales básicas para las cuales no encuentran soluciones } \\
\text { adecuadas ni en el mercado privado ni en las macro-políticas de bienestar. }\end{array}$ \\
\hline Moulaert et al (2013:17) & $\begin{array}{l}\text { La innovación social trata sobre la inclusión social y acerca de contrarrestar y superar las fuerzas } \\
\text { conservadoras que están dispuestas a fortalecer o preservar situaciones de exclusión social. }\end{array}$ \\
\hline $\begin{array}{l}\text { Comisión Europea } \\
(2013)\end{array}$ & $\begin{array}{l}\text { Es el desarrollo e implementación de nuevas ideas (productos, servicios y modelos) para satisfacer las } \\
\text { necesidades sociales, crear nuevas relaciones sociales y ofrecer mejores resultados. Sirve de respuesta } \\
\text { a las demandas sociales que afectan al proceso de interacción social, dirigiéndose a mejorar el bienestar } \\
\text { humano. }\end{array}$ \\
\hline Paz (2015:54) & $\begin{array}{l}\text { Es el resultado de un transcurso de aprendizaje colectivo que nos proporciona soluciones novedosas para } \\
\text { satisfacer demandas y necesidades sociales. }\end{array}$ \\
\hline $\begin{array}{l}\text { World Economic } \\
\text { Forum (2016:5) }\end{array}$ & $\begin{array}{l}\text { Es la aplicación de un enfoque innovador, práctico y sostenible, basado en el mercado para beneficiar a la } \\
\text { sociedad en general, y a las poblaciones de bajos ingresos o desatendidas en especial. }\end{array}$ \\
\hline
\end{tabular}

FUENTE: Elaboración propia a partir de los trabajos citados.

La tabla 1, a la vez que refleja diferentes perspectivas para definir la innovación social, muestra, como indican Djellal y Gallouj (2012), que la expresión innovación social ha resultado problemática desde sus orígenes, ya que, tanto innovación como social, son términos que utilizados en la misma frase crean un nuevo concepto de gran vastedad. Por ese motivo, es interesante realizar un ejercicio de síntesis, y analizar, tal y como hacen Rüede y Lurtz (2012), los diferentes prismas desde los que puede ser entendida. Se permite así identificar tres enfoques principales:

2.- La definición es realizada en uno de sus informes del proyecto europeo "Theoretical, empirical and policy foundations for boulding social innovation in Europe" (TEPSI), en el que participa como socio la Young Foundation.

CIRIEC-España, Revista de Economía Pública, Social y Cooperativa

ISSN: 0213-8093

№ 97/2019, pp. 245-278 
- Innovación social empresarial. Se entiende desde una perspectiva en la que la empresa privada es un elemento central del concepto. Hay una alta referencia a la introducción de novedades en los procesos de gestión, a las mejoras en la organización y a la solución de problemas sociales individuales o colectivos desde la empresa (Pot y Vaas, 2008; Sanchís y Campos 2008; World Economic Forum, 2016). Ésta es la encargada de realizar las innovaciones sociales. Entran dentro de este enfoque prácticas tan variadas como el emprendimiento social, la mejora de condiciones sociales dentro de las empresas, la responsabilidad social o la creación de una producción más sostenible y con menor impacto ambiental.

- Innovación social transformadora. Esta perspectiva hace referencia a la innovación social como elemento de transformación social. Se encuadrarían dentro de ella aquellas actuaciones innovadoras que, ante la ineficiente respuesta del sector público y el mercado, son llevadas a cabo desde la acción colectiva, atendiendo a necesidades y problemas de marcado carácter local (Harris y Albury, 2009; Oosterlynck et al, 2013; Moulaert et al, 2013). También, se resalta la lucha de las comunidades y los movimientos sociales para crear alternativas sociales y mejorar el bienestar social. Esta corriente de pensamiento entiende que los sujetos que llevan a cabo las prácticas socialmente innovadoras son: las empresas sociales ${ }^{3}$, las entidades y organizaciones sin ánimo de lucro, las asociaciones de la sociedad civil y las cooperativas (Mulgan, 2006).

- Innovación social integradora. Este enfoque permite encuadrar ambos casos de iniciativas socialmente innovadoras anteriormente expuestos. Es una solución amplia que surge fruto de la convivencia paralela de las dos perspectivas previamente mencionadas. La innovación social es entendida como un concepto vasto, caracterizado por resolver todo tipo de problemas y necesidades sociales, ofreciendo mejores resultados para toda la sociedad que los existentes hasta el momento. Desde esta perspectiva, se reconoce la existencia de diferentes tipos y grados de innovación social, y se entiende que las iniciativas pueden estar desarrolladas por el sector público, el sector privado o la sociedad civil (NESTA, 2008; Phills et al, 2008; Hubert, 2010; Caulier-Grice et al, 2012; Comisión Europea, 2013).

Por lo tanto, el fenómeno puede ser abordado desde diferentes perspectivas. Nosotros entendemos que la primera está altamente influenciada por la visión más neoliberal, siendo clave la figura del emprendedor social como generador de todo el valor, por lo que tiene más relación con el emprendimiento stricto sensu y con el trabajo por cuenta propia, que con las iniciativas socialmente innovadoras y las comunidades. Por otro lado, el tercer enfoque, en su intento de englobar ambas perspectivas, desvirtúa la innovación social y contribuye a la confusión sobre qué son prácticas socialmente innovadoras. Esto provoca que surjan cada vez más iniciativas que se autodefinen como tal, y que, en cambio, no están en sintonía con lo que entendemos son las características propias del concepto, corriéndose el riesgo de exponerlo a convertirse en un concepto desfigurado y vacío de con-

3.- Las empresas sociales son aquellas que buscan solucionar los problemas sociales básicos de los seres humanos de una manera autosuficiente y rentable, y donde atributos como "sin ánimo de lucro", "público", o "privado" pasan a un segundo lugar para definirlas y clasificarlas. Es una nueva categoría organizativa que permite simultáneamente la creación de valor económico y social (Barrera, 2007: 4). 
tenido propio. Así, consideramos que, por sus propiedades y particularidades, la innovación social se aproxima mejor a la concepción de aquellos autores que la entienden desde el punto de vista transformador.

\subsection{El territorio como el ámbito natural para el desarrollo de la innovación social}

En este segundo subepígrafe vamos a analizar el campo de acción natural de la innovación social, el territorio. Es necesario introducir el componente espacial en el análisis porque la mayoría de las innovaciones sociales se producen y están condicionadas por él (Fontan et al, 2005; MacCallum, 2009; Van Dyck y Van Den Broeck, 2013). Además, existe una amplia evidencia empírica sobre la efectividad de este tipo de innovaciones para lograr un desarrollo territorial más inclusivo y sostenible de las sociedades que residen en ellos (Hillier et al, 2004, Moulaert et al, 2005; Calzada, 2013; Subirats y García-Bernardos, 2015). En este sentido, Subirats (2015) afirma que la innovación social ha supuesto una transformación sobre cómo afrontar y superar los problemas y retos sociales en las ciudades y sus barrios.

Lo primero que debemos entender cuando hablamos de innovación social en el territorio es qué es "el territorio". Méndez (2002) señala que éste no es una simple proyección espacial, sino una construcción social, reflejo de acciones y comportamientos múltiples, acumulados en el tiempo, con capacidad de influir también de forma significativa sobre la estructura y funcionamiento de la sociedad. En consecuencia, nuestros territorios están directamente determinados por el modelo neoliberal predominante; el mercado asigna bienes y servicios de la forma más eficiente posible y el sector público corrige los fallos que éste genera.

Como resultado de ello, las dinámicas de desarrollo de los territorios y las sociedades que viven en ellas se sitúan en este contexto, y es evidente observando la realidad, que este sistema no es totalmente inclusivo ya que hay personas que son marginadas (Moulaert et al, 2007; Blanco, 2018). Por ese motivo, Hillier et al (2004) defienden que se hace cada vez más necesario utilizar mecanismos y herramientas socialmente innovadoras que coadyuven a integrar a aquellos que han quedado excluidos por el funcionamiento propio del sistema hegemónico.

La innovación social es especialmente interesante de analizar en las ciudades y barrios, ya que son los espacios donde la mayoría de la sociedad vive y donde se reflejan de forma más evidente los problemas urbanos (pobreza, desempleo, dificultad de acceso a la vivienda, gentrificación, contaminación, entre otros). Estas características los convierten en los lugares idóneos para desarrollar procesos de transformación social (Subirats, 2015 y Blanco, 2018).

Con referencia a esto último, afirman Moulaert et al (2004) que establecer estrategias de desarrollo urbano basándose en la innovación social es de gran utilidad para lograr cambios de gobernanza en las comunidades locales. A pesar de ello, nos recuerda Subirats (2015: 96) que, aun cuando se 
consigan esas ansiadas transformaciones en la gobernanza, hay que tener en cuenta que los gobiernos locales ocupan "una posición periférica en un entramado de gobierno multinivel" y tienen unos recursos que en ocasiones resultan insuficientes para afrontar problemas de gran magnitud.

Lo cierto es que, aunque la efectividad final de la innovación social para lograr un cambio radical puede ser limitada, su inclusión en la gobernanza local se postula como una solución para reducir el impacto de poderosos grupos de interés en las estrategias territoriales que marcan el devenir de los espacios urbanos. La aprobación de políticas urbanas es fundamental que represente el interés de la mayoría social, y no el particular de un grupo o colectivo (Caravaca, 2018). Por esa razón, una de las principales motivaciones de las comunidades socialmente innovadores debe ser ejercer, a través de sus iniciativas, un mayor control en la toma de decisiones que afectan a las sociedades donde habi$\tan$ (Moulaert et al, 2004).

No obstante, las mencionadas comunidades deben estar vertebradas mediante redes que les permitan estar interconectadas para que puedan desarrollar un auténtico cambio territorial a través de nuevas fórmulas (Moulaert et al, 2004). A este respecto, según Belda-Miquel (2018) urge acabar con la hegemonía de la innovación top-down y promover innovaciones desde las comunidades con enfoques bottom-up. Esto, sugiere Navarro (2015), es fundamental para reintroducir la inclusión social como eje para un desarrollo local más sostenible.

Por su parte, Caravaca (2018) plantea que actualmente existen alternativas para la revitalización de los espacios urbanos en los territorios que se basan en los recursos de proximidad y en la existencia de redes de colaboración, siendo el objetivo principal romper con las estructuras que predominan en el sistema. Concibe esta autora que las prácticas dinamizadoras de los espacios urbanos van desde la producción de bienes o servicios, hasta la financiación, el consumo o el intercambio.

Por último, hay que subrayar que la introducción del elemento territorial en el análisis es primordial para comprender qué factores son determinantes para el desarrollo de la innovación social. Ésta tiene un fuerte componente local por su potencial para crear procesos y nuevas lógicas de actuación que permiten generar efectos positivos en las ciudades y barrios, reduciendo la polarización social. Por lo tanto, su estudio en el territorio tiene mucha más relación con el enfoque que la entiende desde el punto de vista transformador, que con los otros anteriormente expuestos.

\subsection{Factores determinantes para la irrupción de la innovación social}

Al igual que sucede para cualquier proceso de innovación tecnológica, el desarrollo de la innovación social no puede ser diseñado en su integridad. Sin embargo, se pueden planificar estrategias que permitan aumentar la probabilidad de que la innovación tenga lugar (Chesbrough, 2006; Vrontis et al., 2012). Así pues, de forma mimética a como ocurre para la innovación tecnológica, si la innovación social no puede programarse, es primordial implementar un ecosistema ${ }^{4}$ que permita aumentar sus 
posibilidades de aparición. De esta forma, la identificación de un contexto propicio para que emerjan iniciativas socialmente innovadoras resulta muy relevante para proporcionar conocimiento a aquellos que deseen fomentar y promover un modelo socioeconómico más sostenible basado en este tipo de prácticas (Caulier-Grice et al, 2012; Bureau of European Policy Advisers, 2014; Neumeier, 2017).

En una visión amplia y general sobre los factores que son claves para el desarrollo de la innovación social en un territorio, Neumeier (2017) señala tres:

1. Determinantes y variables que son importantes para el éxito de cualquier tipo de innovación. La irrupción y proliferación de innovaciones (tecnológicas, organizacionales...) necesita también de un marco que permita su desarrollo. Por ello, el autor referido, indica que hay una serie de factores que son necesarios y comunes para cualquier tipo de innovación.

2. Determinantes que posibilitan o dificultan las actuaciones de los agentes socialmente innovadores. Por ejemplo, factores como la facilidad de financiación o de obtener fondos, las estructuras sociales, la base jurídica y legal y la calidad de la regulación, entre otros.

3. Determinantes que influyen en el proceso participativo subyacente en la innovación social. La creación de redes, el apoyo institucional o la educación son muy valiosos para fomentar la participación.

Otros factores que son sugeridos en la bibliografía como transcendentes para la generación de las innovaciones sociales son: el tamaño de la región (Abreu y Cruz, 2011; Oosterlynck et al, 2013), el contexto cultural (Echeverría y Gurrutxaga, 2012; Hernández-Ascanio et al, 2016), la existencia de seguridad jurídica (Howaldt et al, 2016), la concesión de subvenciones (Bureau of European Policy Advisers, 2014), la existencia de cohesión (Krlev et al, 2014), la capacidad de absorción de conocimiento (Unceta et al, 2016: 193; Bernardino y Santos, 2017: 162) o el uso de herramientas digitales y la tecnología (Mulgan, 2006; Bureau of European Policy Advisers, 2014).

Con ello se evidencia que el análisis de por qué irrumpe en unos territorios la innovación social más que en otros, no viene únicamente condicionado por el grado de pobreza o las necesidades insatisfechas, sino que depende de todo un conjunto de factores determinados por la trayectoria socioeconómica del territorio.

A este respecto, Blanco et al (2016) afirman que, paradójicamente, no es en las regiones donde existen más necesidades y problemas sociales donde la generación de iniciativas socialmente innovadoras es mayor, sino en aquellas donde concurren una serie de condiciones favorables para ello.

4.- Ferraris y Greco (2015) afirman que el concepto general de ecosistema de innovación se refiere al "conjunto de personas, instituciones, políticas y recursos que promueven el traspaso de nuevas ideas en productos y procesos". 
Por consiguiente, sin dejar de reconocer el valor que pueden tener factores como la presencia de necesidades insatisfechas para el nacimiento de innovaciones sociales, hay que considerar como requisito indispensable lograr que existan en el territorio una serie de elementos que permitan fomentar su desarrollo.

\section{Notas metodológicas}

En este trabajo se han utilizado una serie de técnicas de análisis cualitativas. El estudio de la innovación social como concepto y de los elementos determinantes para su aparición ha sido llevado a cabo a partir de la revisión del estado del arte y la realización de entrevistas semiestructuradas.

Con respecto a la revisión de la literatura, que ha sido realizada en el anterior apartado, son dos las fases que pueden ser distinguidas.

- La primera fase, en la que se realiza el análisis del estado de la cuestión, centrando nuestra atención en las diferentes corrientes de pensamiento y en la significatividad del componente espacial.

- La segunda fase, en la que se lleva a cabo un análisis de los factores que influyen en la creación de un entorno favorable para el nacimiento de prácticas socialmente innovadoras.

Teniendo en cuenta la revisión del estado del arte, y siendo conocedores de la problemática, hemos diseñado una metodología a partir de entrevistas semiestructuradas. La hemos elegido por entender que, aunque existe una amplia y valiosa literatura, la innovación social es un fenómeno vivo y en constante evolución. Esto hace que sea de gran utilidad confrontar las ideas evidenciadas tras la revisión de una manera pragmática. La información procedente de las entrevistas nos permite aportar, de forma empírica y fidedigna, nuevos elementos claves tanto para definir la innovación social, como para reconocer y ponderar la existencia de nuevos factores esenciales para su aparición.

\subsection{Entrevistas}

La identificación de los elementos más considerables que han influido en la aparición de las innovaciones sociales desde una perspectiva territorial ha sido realizada teniendo en cuenta la visión de tres grupos previamente definidos 5 : agentes que han desarrollado iniciativas socialmente innovado- 
ras como Ana Hevilla, que es la responsable del Área Social del Grupo de Desarrollo Rural Valle del Guadalhorce; agentes de centros de fomento de la innovación social como David Pino, que es director de Innovación y Economía Social del Ayuntamiento de Sevilla; científicos y académicos como Pilar Tirado, que ha publicado trabajos de impacto en materia de innovación social. Estos tres perfiles, que son diferentes pero complementarios, nos permiten tener una visión completa y transversal acerca del fenómeno.

Con respecto a la selección de los entrevistados, hay que mencionar que se ha primado fundamentalmente la relevancia de la experiencia y del proyecto, la trayectoria académica y profesional, así como los reconocimientos y premios que organismos de prestigio han otorgado en materia de innovación social a los individuos, o a los colectivos que representan, por su buen hacer6.

Teniendo siempre en cuenta los mencionados criterios hemos tratado de contactar con un total de 87 personas, siendo 24 las que finalmente accedieron a realizar las entrevistas. En la selección de los sujetos también existió un cierto grado de oportunidad que el propio avance de la investigación propició, ya que los mismos entrevistados nos facilitaron en ocasiones la posibilidad de contactar con expertos y perfiles interesantes que cumplían con los criterios establecidos. Las entrevistas fueron realizadas entre los meses de junio y noviembre de 2018 y tuvieron una duración de entre 50 y 75 minutos.

La estructura seguida para la realización de las entrevistas consta de tres partes diseñadas para conseguir los objetivos de la investigación; la primera, en la que se planteaban preguntas genéricas sobre innovación social; la segunda, en la que se cuestionaba acerca de los aspectos más significativos de su proceso de generación y, por último, una sección dedicada a los efectos y resultados a los que, en opinión de los entrevistados, la innovación social puede dar lugar?

\subsection{Tratamiento de los datos}

Con el objetivo de analizar el contenido de las 24 entrevistas realizadas se ha utilizado el programa informático Atlas.ti. Siguiendo a Prados (2007:62) "Atlas.ti es una herramienta informática la cual es capaz de analizar datos cualitativos complejos de forma estructurada (...) que se articula alrededor de la Grounded Theory, y que trata de generar teoría a la vez que investiga en la materia; ambas como parte de un mismo proceso". Esta herramienta nos ha asistido en el análisis del contenido ${ }^{8}$ de las transcripciones, facilitando nuestro posterior trabajo interpretativo.

6.- En todos los casos se han seleccionado responsables de organizaciones con una amplia trayectoria, responsables directos de los proyectos, centros o empresas, así como académicos y científicos con publicaciones de alto impacto sobre la materia.

7.- En el anexo 2 puede ser consultado el guion seguido para realizar las entrevistas semiestructuradas.

8.- Siguiendo a Berelson (1952) el análisis de contenido es una técnica de investigación que busca reducir la subjetividad del investigador y ser más sistemática y cuantitativa. 
El análisis de contenido permite descubrir componentes básicos de un fenómeno concreto teniendo en consideración las ideas expresadas por los entrevistados a partir de palabras y frases (Noguero, 2002). A este respecto, afirma Escalante (2009), que dichas expresiones y la trascendencia de los conceptos presentes en un texto son de elevada significatividad, ya que a partir de ellos podemos identificar su importancia y relación.

Nosotros, para la exploración e interpretación de los textos hemos analizado los contextos lingüísticos y semánticos de aquellas palabras o frases que eran susceptibles de expresar un concepto. En total hemos realizado un análisis de 24 archivos primarios, que representan cada una de las transcripciones de las entrevistas realizadas.

Lo primero que hemos llevado a cabo tras la transcripción de las entrevistas es su codificación. Este proceso supone una forma de reducción de datos, ya que, partiendo de un gran volumen de información, logramos segmentar ciertos fragmentos de la información (citas) y etiquetarlas a partir de códigos (Pérez-López et al, 2015). Estos son los elementos principales de análisis y son considerados como un sistema de categorías. En nuestro caso, cada vez que hemos identificado una cita, que hacía referencia a la existencia de un factor que condicionaba la irrupción de la innovación social o que enunciaba características que la definían, hemos etiquetado un código -referido a un determinado factor relacionado con el contenido de la cita9 ${ }^{9}$. Por consiguiente, la codificación ha contribuido a realizar conexiones entre las diferentes partes de la información que disponemos.

Una vez realizada la codificación, cuando ya teníamos todos los códigos, los hemos organizados en familias ${ }^{10}$. Concretamente, en sintonía con los fines principales de nuestro análisis, en dos familias: una, en la que se recogen los códigos relativos a la definición de innovación social, y otra, relacionada con los factores determinantes. Además, hay que destacar que el programa Atlas.ti cuenta con la herramienta WordCruncher para contar la frecuencia absoluta de los códigos etiquetados ${ }^{11}$. Señala Noguero (2002) que dichas frecuencias de aparición de los códigos son esenciales para realizar un análisis estadístico del contenido.

Una vez conocidas las frecuencias absolutas de los códigos, para obtener más información acerca de la importancia de cada uno de ellos, era necesario relativizarlos. Para dicho fin hemos considerado que los códigos previamente habían sido divididos por familias: por un lado, se han sumado todos los códigos que definían la innovación social ${ }^{12}$ y, por otro lado, de los que determinan los factores relevantes para su aparición ${ }^{13}$.

9.- En una cita pueden identificarse uno o más factores, lo que hace que se puede etiquetar dicha cita con uno o más códigos. Cuando en una cita se etiquetan más de un código, estos coocurren, lo que es signo evidente de que puede existir una relación entre los factores a los que representan.

10.- Así como los códigos "agrupan" citas, es posible crear agrupaciones de los códigos mediante familias.

11.- Las frecuencias absolutas de los códigos hacen referencia al número de veces que una cita ha sido identificada con un mismo código. Por lo tanto, la frecuencia absoluta nos da información acerca del número de veces que en el contenido del texto transcrito se ha hecho referencia a un factor determinado.

12. - En total se han etiquetado 212 veces códigos englobados en la familia definición de la innovación social.

13. - En total se han etiquetado 647 veces códigos englobados en la familia factores relevantes para su aparición. 
Teniendo en cuenta dichos sumatorios, hemos podido calcular la frecuencia relativa ${ }^{14}$ de cada uno de los códigos por familias, lo que contribuye a conocer qué factores, de los que los entrevistados han señalado, tienen más fuerza en la aparición de la innovación social ${ }^{15}$.

Una vez identificados los códigos y sus frecuencias, se generaron redes de coocurrencia ${ }^{16}$, que permitieron analizar las diferentes interacciones entre cada uno de los códigos señalados. Así se ha facilitado la agrupación de los factores por temáticas, creando cinco grandes pilares que engloban a cada uno de ellos.

\section{Discusión de los resultados}

Tras la revisión del estado del arte, y teniendo en consideración la frecuencia relativa de los códigos que representaban características de la innovación social, encuadrados en la familia definición de la innovación social, planteamos una propuesta de definición: Prácticas o iniciativas llevadas a cabo por la comunidad que, a partir de los productos, servicios o modelos que generan, o bien a través del proceso diseñado para conseguir sus objetivos, alcanzan soluciones que dan una mejor respuesta, de forma alternativa y creativa, a problemas o necesidades sociales.

Esta definición recoge nuestra forma de entender la innovación social tras la profunda revisión de la literatura realizada y los conocimientos que los expertos entrevistados nos han aportado. Son para nosotros postulados esenciales:

- Que la innovación social tenga, como sujeto social y político principal, a la comunidad. Primamos el elemento colectivo por encima del individual.

- Que el objetivo sea la búsqueda de respuestas, no necesariamente novedosas o innovadoras, sino alternativas. A partir de ellas, se deben alcanzar soluciones a problemas sociales que afectan al territorio.

- La existencia de un elemento rupturista con respecto a la hegemonía de funcionamiento del mercado tradicional.

Una vez realizada la definición, en la tabla 2, se presentan los cinco pilares que facilitan que se origine: elementos sociales y culturales; apoyo político e institucional; conocimiento y mecanismos facilitadores; componentes espaciales; entidades y mecanismos que determinan la estructura productiva empresarial y social. Los pilares están formados en total por 29 factores.

14.- La frecuencia relativa de los códigos hace referencia al número de veces que se repite un código (n) en una familia con respecto número total de veces que aparecen códigos englobados en esa familia. Por ejemplo, para la familia factores relevantes para su aparición la frecuencia relativa de un código cualquiera sería $=577$. Puede comprobarse dicho cálculo a partir de la tabla 2.

15. - López et al (2015) y Fraile et al (2018), señalan que este estadístico descriptivo, ayuda a entender la fuerza con la que subyace un factor en el fenómeno investigado.

16.- La coocurrencia hace referencia a aquellos códigos que tienen una relación en el sentido de que coocurren en alguna cita. Ver $n$. 9. 


\section{Tabla 2. Factores ponderados que determinan la aparición de la innovación social en los territorios}

\begin{tabular}{|c|c|c|c|c|c|}
\hline $\begin{array}{l}\text { Elementos sociales y culturales } \\
\text { de la población }\end{array}$ & $\begin{array}{l}\text { Frecuencia } \\
\text { absoluta }\end{array}$ & $\begin{array}{l}\text { Frecuencia } \\
\text { relativa }\end{array}$ & Componentes espaciales & $\begin{array}{l}\text { Frecuencia } \\
\text { absoluta }\end{array}$ & $\begin{array}{l}\text { Frecuencia } \\
\text { relativa }\end{array}$ \\
\hline Existencia de preocupación social & 33 & 0,05719 & Nivel socioeconómico de la población & 55 & 0,09532 \\
\hline Propensión cultural al cambio & 33 & 0,05719 & Densidad de la población & 9 & 0,01559 \\
\hline Propensión a la participación & 23 & 0,03986 & Orografía & 6 & 0,01039 \\
\hline Propensión a la colaboración & 22 & 0,03812 & Posición geográfica & 6 & 0,01039 \\
\hline $\begin{array}{l}\text { Existencia de una clase creativa } \\
\text { Capacidad y disponibilidad para }\end{array}$ & 21 & 0,03638 & $\begin{array}{l}\text { Existencia de recursos naturales próximos } \\
\text { Existencia de otras experiencias socialmente }\end{array}$ & 3 & 0,00519 \\
\hline $\begin{array}{l}\text { trabajar con diferentes agentes } \\
\text { económicos y generar acción colectiva } \\
\text { Existencia de una cultura asociativa } \\
\text { de los individuos }\end{array}$ & a & 0,02946 & innovadoras & 3 & 0,00519 \\
\hline Totales & 161 & 0,27902 & Totales & 82 & 0,14211 \\
\hline Apoyo político e institucional & $\begin{array}{c}\text { Frecuencia } \\
\text { absoluta }\end{array}$ & $\begin{array}{l}\text { Frecuencia } \\
\text { relativa }\end{array}$ & $\begin{array}{l}\text { Entidades y mecanismos que determinan } \\
\text { la estructura productiva empresarial y social }\end{array}$ & $\begin{array}{l}\text { Frecuencia } \\
\text { absoluta }\end{array}$ & $\begin{array}{l}\text { Frecuencia } \\
\text { relativa }\end{array}$ \\
\hline $\begin{array}{l}\text { Existencia de programas de apoyo } \\
\text { a la innovación social }\end{array}$ & 51 & 0,08838 & $\begin{array}{l}\text { Nexos que favorecen las relaciones } \\
\text { público-privadas }\end{array}$ & 25 & 0,04332 \\
\hline $\begin{array}{l}\text { Existencia de laboratorios o centros } \\
\text { de innovación social }\end{array}$ & 34 & 0,05892 & $\begin{array}{l}\text { Existencia de cooperativas } \\
\text { Existencia de entidades sociales }\end{array}$ & $\begin{array}{l}23 \\
11\end{array}$ & $\begin{array}{l}0,03986 \\
0,01905\end{array}$ \\
\hline Existencia de financiación pública & 34 & 0,05892 & Actividad empresarial activa. Emprendimiento & 6 & 0,01039 \\
\hline $\begin{array}{l}\text { Existencia de una regulación } \\
\text { eficiente y ausencia de trabas }\end{array}$ & 17 & 0,02946 & $\begin{array}{l}\text { Reconocimiento y existencia de premios } \\
\text { (favorece la visibilización) }\end{array}$ & 5 & 0,00866 \\
\hline $\begin{array}{l}\text { Políticas de educación para fomentar } \\
\text { la innovación }\end{array}$ & 12 & 0,02079 & & & \\
\hline Totales & 148 & 0,25649 & Totales & 70 & 0,12131 \\
\hline $\begin{array}{l}\text { Conocimientos y mecanismos } \\
\text { facilitadores }\end{array}$ & $\begin{array}{c}\text { Frecuencia } \\
\text { absoluta }\end{array}$ & $\begin{array}{l}\text { Frecuencia } \\
\text { relativa }\end{array}$ & & & \\
\hline $\begin{array}{l}\text { Conocimiento de la realidad local } \\
\text { Conocimiento de las oportunidades y } \\
\text { potencialidades de nuevos medios }\end{array}$ & 32 & 0,05545 & & & \\
\hline $\begin{array}{l}\text { e instrumentos } \\
\text { Facilidades y medios para establecer }\end{array}$ & 26 & 0,04506 & & & \\
\hline $\begin{array}{l}\text { nuevas comunicaciones y redes } \\
\text { Espacios para el desarrollo de las }\end{array}$ & 22 & 0,03812 & & & \\
\hline iniciativas & 17 & 0,02946 & & & \\
\hline $\begin{array}{l}\text { Presencia de otros tipos de innovación } \\
\text { Mecanismos de cooperación entre }\end{array}$ & 10 & 0,01733 & & & \\
\hline universidad-ciencia-sociedad & 9 & 0,01559 & & & \\
\hline Totales & 116 & 0,20103 & & & \\
\hline
\end{tabular}

FUENTE: Elaboración propia a partir del análisis de las entrevistas con Atlas.ti. 


\subsection{Análisis de los factores y sus relaciones}

En la tabla 2, además de los factores que son determinantes de la innovación social y los pilares en los que por temática se agrupan, se han mostrado las frecuencias absolutas y relativas de cada uno de ellos. Dichas frecuencias son utilizadas en este trabajo como proxy para ponderar la importancia de los factores y pilares señalados. Esto permite conocer cuáles son más necesarios y tienen mayor peso en la facilitación de la aparición de las innovaciones sociales.

Los diez factores con mayor impacto son: el nivel socioeconómico de la población (0.09532), la existencia de estrategias y líneas de actuación para desarrollar la innovación social (0.08838), la existencia de laboratorios o centros de innovación social $(0.05892)$, la existencia de financiación pública (0.05892), la existencia de preocupación social (0.05719), la propensión cultural al cambio $(0.05719)$, el conocimiento de la realidad local (0.05545), el conocimiento de las oportunidades y potencialidades de nuevos medios e instrumentos ( 0.04506$)$, los nexos que favorecen las relaciones público-privadas (0.04332) y la existencia de cooperativas (0.03986).

Por lo tanto, a partir de la tabla 2, se aporta conocimiento que puede ser útil para mejorar la elaboración de políticas de innovación social a partir del desarrollo de estrategias que permitan priorizar los elementos a fomentar, y tengan en cuenta, no sólo qué factores influyen en su irrupción, sino cómo de relevantes son cada uno de ellos. A continuación, pasamos a explicar, por pilares, cada uno de sus componentes por orden de significatividad relativa.

\subsubsection{Elementos sociales y culturales de la población}

El primer pilar es elementos sociales y culturales de la población y hemos agrupado un total de siete factores.

Entre esos elementos, la existencia de preocupación social y la propensión cultural al cambio se presentan, con la misma frecuencia relativa, como los factores más relevantes en este pilar.

La existencia de preocupación social ante ciertos problemas hace germinar acciones que buscan resolver total o parcialmente dichos retos. Pese a ello, ese motor se ve en ocasiones limitado si no existe una tolerancia y propensión a los cambios por parte de la población. Por ese motivo, la propensión cultural al cambio aparece como la primera barrera o limitación que existe en cualquier sociedad. Si pensamos que las cosas no se pueden cambiar, que vienen ya predeterminadas, o que de manera individual se tiene una escasa capacidad de transformación, las posibilidades de que surjan iniciativas que logren penetrar en la sociedad son muy bajas. En consecuencia, la resistencia a actuar de forma distinta y la aversión al riesgo son situaciones que deber ser combatidas fomentando una metamorfosis cultural de la población. 
El tercer y cuarto factor hacen referencia a participary colaborar en aquellos asuntos que dan origen tanto a preocupaciones sociales como personales. A través de la participación, los sujetos conocen a otras personas, entendiendo que hay muchas de éstas que tienen sus mismas motivaciones e inquietudes, lo cual fortalece su espíritu y reduce su animadversión a enfrentarse a nuevas situaciones. Los individuos entienden que, a través de la colaboración, se puede lograr la consecución de metas que de forma individual parecían inalcanzables.

Todo ello se ve favorecido por el que es el quinto factor en este pilar; la existencia de una clase creativa. Los retos globales y complejos requieren de personas creativas que diseñen soluciones alternativas. La creatividad es una característica necesaria para el diseño de dichas soluciones, que serán más efectivas, cuando en su elaboración colaboren tanto grupos de interés cercanos como con otros agentes económicos y sociales. Por consiguiente, el sexto factor relevante es la capacidad y disponibilidad para trabajar con todos los agentes económicos y generar acción colectiva.

Estos entornos creativos donde individuos muy diferentes se unen para buscar soluciones a sus problemas, se originan con mayor facilidad en aquellos territorios donde existe una cultura asociativa fuerte. A pesar de que este factor es el que tiene un menor peso en el pilar, hay que tenerlo en cuenta ya que el sentimiento de unión hace que las asociaciones actúen en muchos casos como nodos para la innovación social, facilitando la acción colectiva y de la comunidad.

\subsubsection{Apoyo político e institucional}

El segundo pilar clave es el apoyo político e institucional. Se encuadran en éste un total de cinco factores y se evidencia mediante él la necesidad de elaborar líneas de actuación concretas desde aquellas administraciones públicas que deseen fomentar las experiencias socialmente innovadoras.

En este sentido, aunque la innovación social suele germinar desde procesos bottom-up, su nacimiento y desarrollo se encuentran muy condicionados a que desde la esfera pública se apliquen las políticas y medidas adecuadas. Este apoyo se canaliza, en muchas ocasiones, en la elaboración de un programa en el que se detallen una batería de acciones y líneas de actuación que contribuyan a la aparición de iniciativas socialmente innovadoras -teniendo en cuenta las carencias que hay en los territorios-. Por ello, el factor más significativo en este pilar es la existencia de programas de apoyo a la innovación social.

Sin embargo, la innovación social no debe ser institucionalizada si lo que se pretende es lograr los efectos positivos que se derivan del fenómeno. Cuando desde las instituciones se intenta diseñarla, se corre el riesgo de perder aquellas características que aportan su auténtico valor añadido. Los planes y programas deben cubrir aquellas necesidades que las comunidades y las iniciativas socialmente innovadoras de cada territorio presenten, así pues, es primordial que se realicen de forma conjunta con los encargados y responsables de dichas iniciativas. 
Entre las medidas que desde la administración se suelen llevar a cabo destaca la creación de centros o laboratorios de innovación social. Este factor es el segundo más relevante en este pilar. La utilidad de dichos centros o laboratorios reside en su capacidad para asesorar, aportar medios y aumentar el impacto y la probabilidad de éxito de las iniciativas socialmente innovadoras. Además, son un punto de encuentro ideal para colaborar y lograr sinergias con otras iniciativas y comunidades, así como para estar en contacto permanente con los decisores públicos.

Por otro lado, el tercer factor (que tiene la misma frecuencia relativa que el anterior mencionado) hace referencia a que desde los organismos públicos se facilite la posibilidad de acceder a financiación pública. Hay que considerar que los proyectos socialmente innovadores suelen tener muchas dificultades para conseguir financiación en el mercado. Esto puede provocar que, pese que las iniciativas deban aspirar a autofinanciarse, en las primeras etapas de su nacimiento sea necesario apoyarlas económicamente hasta su consolidación.

El cuarto factor es que desde la esfera pública se logre crear una regulación eficiente y con cierto grado de flexibilidad en la que no exista burocracia innecesaria. El papel que la legislación tiene es esencial en la creación de un ecosistema beneficioso para la innovación social. Las iniciativas sufren en ocasiones dificultades para emerger por su multidisciplinariedad y transversalidad, lo que no está en consonancia con las regulaciones tradicionales diseñadas, en su mayoría, de forma sectorial. Así pues, adaptar la regulación es trascendental para que no se convierta en un problema sistémico. Además, la reducción de trabas, garantizar la unidad de mercado y plantear ciertas ventajas fiscales son otras medidas que tendrían un impacto muy positivo.

El último factor es la idoneidad de que existan acciones y políticas públicas que fomenten una cultura de la innovación desde la infancia. De esta manera, crear un tejido o un entorno que favorezca la innovación social es mucho más plausible, ya que, desde una edad muy temprana, se habría motivado y enseñado que la innovación es una herramienta útil para conseguir transformar situaciones estructurales.

\subsubsection{Conocimientos y mecanismos facilitadores}

El tercer pilar es conocimientos y mecanismos facilitadores y hemos agrupado un total de seis factores que facilitan crear un entorno favorable a la innovación social. El factor con mayor peso dentro de este pilar es el conocimiento de la realidad local. Hace referencia al conocimiento del entorno, de sus problemas y de su casuística. La mejor manera de diseñar una metodología para resolver un problema social es saber en profundidad acerca de éste y conocer los medios con los que se cuentan para poder solucionarlo.

A este respecto, el segundo factor es el conocimiento de las oportunidades y potencialidades de nuevos medios e instrumentos como el co-dising o el crowfunding, nuevos espacios de trabajo como los coworking o nuevos modelos que rompen estructuralmente con la hegemonía del sistema capi- 
talista como el co-housing. La familiarización con modelos y nuevas fórmulas de gestión existentes, junto al conocimiento de la realidad local, es un binomio que genera soluciones socialmente innovadoras.

Otro factor valioso es la existencia de facilidades y medios para establecer nuevas comunicaciones y redes que posibiliten la puesta en común de ideas y la retroalimentación. Los medios pueden ser foros, congresos o reuniones multilaterales que facilitan el aprendizaje de nuevas técnicas y aplicaciones para mejorar la eficiencia de las respuestas.

El cuarto factor más determinante en este pilar es la posibilidad de disponer de un espacio. El tener un espacio, ya sea físico o no, para el desarrollo de la actividad es una forma de estabilización y consolidación para las iniciativas. También es un elemento que guarda una alta relación con el factor anteriormente mencionado ya que los espacios concretos facilitan y fomentan las relaciones sociales, funcionando como polos de atracción y publicidad de las iniciativas.

Por otro lado, el quinto factor es la presencia de otros tipos de innovación en el territorio. Esto beneficia al dinamismo social y repercute en las facilidades para crear iniciativas socialmente innovadoras por un doble motivo: en primer lugar, porque éstas pueden nutrirse de otras innovaciones, y, en segundo lugar, porque allí donde hay innovaciones de cualquier tipo significa que existen una serie de factores que pueden ser comunes y necesarios para otras.

Finalmente, cabe especificar que, aunque todos los factores tienen una elevada importancia, todos ellos se verían reforzados -y los proyectos ganarían más fuerza- con metodologías y estudios científicos que las analizasen y apoyasen. Por ello, que existan mecanismos que permitan la transferencia de conocimiento desde la universidad y centros de investigación a la sociedad, es un elemento que coadyuvaría a fortalecer el deseable ecosistema. Éste se postula como el último factor dentro de este pilar.

\subsubsection{Componentes espaciales}

El cuarto pilar hace referencia a un conjunto de seis factores que se encuadran bajo la denominación de componentes espaciales. En este pilar se agrupan aquellos que determinan al territorio stricto sensu, pero, debe aclararse, que como hemos expuesto en anteriores epígrafes, el territorio afecta y condiciona de forma transversal a todos ellos.

A este respecto, el factor más determinante tanto de este pilar como de todos los señalados en la tabla 2 es el nivel socioeconómico de la población. Es el factor con mayor peso (0.09532) debido a que hace alusión a variables tan significativas para la caracterización del espacio como el empleo, los ingresos 0 el nivel de educación. Son todos ellos condicionantes de las capacidades sociales y económicas de los territorios, lo que afecta a las potencialidades y necesidades de los individuos para desarrollar acciones que aumenten su bienestar social. 
En este sentido, una sociedad con nivel socioeconómico medio será mucho más propensa a generar innovaciones sociales que una sociedad con un nivel socio económico alto o bajo. Muestra de ello es que, en los barrios con nivel socioeconómico más alto, donde las personas pueden cubrir sus necesidades acudiendo al mercado, no existen -salvo excepciones- muchas iniciativas socialmente innovadoras. En el polo opuesto, en los barrios con nivel socioeconómico más bajo, ocurre que hay necesidad de innovación social ya que, ni el mercado ni la administración pública satisfacen las carencias sociales existentes, pero, en cambio, la capacidad de estas personas para lograr poner en marcha este tipo de soluciones bottom-up es limitada. Por consiguiente, la innovación social necesita de una población con un nivel socioeconómico medio, que tengan necesidades insatisfechas y tengan capacidad para generar iniciativas a partir de la acción colectiva (Blanco et al, 2016).

El segundo elemento en este pilar es la densidad de población. La innovación social tiende a irrumpir más en áreas urbanas muy pobladas, donde los problemas más acuciantes suelen estar relacionados con empleo, vivienda, transporte y contaminación, entre otros. La existencia de situaciones complejas, relacionadas con estas materias debido a la alta densidad poblacional, acarrea la necesidad de buscar soluciones alternativas y creativas.

Sin embargo, la innovación social puede verse también impulsada por las condiciones propias de las zonas rurales que suelen tener bajos niveles de densidad de población. Esto sucede en estas áreas por los retos existentes como la despoblación, el envejecimiento o las pocas oportunidades laborales. Este contexto hace que surjan iniciativas socialmente innovadoras que tratan de cambiar la realidad de su entorno. Aun así, no siempre es sencillo porque muchas zonas rurales se ven muy limitadas por su situación geográfica. La posición del territorio y su orografía son el tercer y cuarto factor en este pilar -ambos con una misma frecuencia relativa- ya que estos pueden suponer una oportunidad o una limitación para el desarrollo de innovaciones sociales. No obstante, por lo general, aquellas zonas que se encuentran cercanas a núcleos económicos activos y que no están aisladas o difícilmente accesibles por la orografía, suelen ser un mejor caldo de cultivo para que las innovaciones sociales emerjan.

El quinto factor de este pilar es la existencia de recursos naturales de proximidad. En aquellos territorios con recursos suelen surgir iniciativas para potenciar su uso y consumo. Son muchas las iniciativas que han aprovechado los recursos y oportunidades que su enclave geográfico les brindaba para contribuir a la inclusión y a la integración social. Por último, hay que destacar, que las iniciativas se ven muy beneficiadas cuando coexisten en el territorio con otras experiencias de innovación social tanto por las sinergias y las nuevas oportunidades que se pueden generar, como por el efecto llamada que para otras personas y colectivos pueden tener. Por lo tanto, la presencia de otras iniciativas se considera el último factor a tener en cuenta en este pilar. 


\subsubsection{Entidades y mecanismos que determinan la estructura productiva empresarial y social}

El pilar quinto está compuesto por cinco factores y ha sido denominado entidades y mecanismos que determinan la estructura productiva empresarial y social. Este pilar engloba al tipo de agentes económicos y sociales y a los mecanismos que favorecen la irrupción de la innovación social.

El factor con mayor peso en este pilar es la existencia de nexos que facilitan las relaciones entre la sociedad civil, la administración pública y el sector privado. Este elemento permite enriquecer ideas y proyectos, generando actuaciones y líneas muy potentes derivadas del consenso de todas las partes. Un ejemplo son las mesas para la mejora de la regulación que permiten que, tanto la sociedad civil como el sector industrial, estén representados y puedan coadyuvar a lograr que el marco que define la estructura empresarial y social refleje sus necesidades.

Por otro lado, el segundo factor hace referencia a la tipología de empresas que tienen un impacto positivo en el desarrollo de las iniciativas socialmente innovadoras; la existencia de cooperativas. Éstas son catalizadoras de la innovación social ya que poseen características que fomentan la democratización de los procesos, la toma de decisiones y el valor social.

Algo similar a lo que ocurre con las cooperativas sucede con las entidades sociales. La existencia de este tipo empresas es el tercer factor con más peso en este pilar. Estas entidades tienen una motivación social y les urge buscar soluciones novedosas a problemas sociales persistentes. Por ello, se encuentran en constante búsqueda de nuevos modelos o prácticas que les permitan mejorar sus actuaciones y aumentar el bienestar del colectivo al que se dirigen.

El cuarto factor, por orden de significatividad, es la existencia de actividad empresarial y emprendimiento en el territorio. Es un indicador claro de las inquietudes y dinamismo de los individuos, pero es también principalmente útil porque cuando el emprendimiento es elevado, a menudo se pueden crear iniciativas conjuntas que promuevan la transformación social. Por ello, es valiosa la presencia de emprendedores sociales que, aunque no hayan creado comunidades para la búsqueda de soluciones, tienen como objetivo diseñarlas para conseguir resolver un reto o mejorar el bienestar de un colectivo a partir de su propia capacidad. El emprendedor social es un aliado natural de las comunidades socialmente innovadoras.

Finalmente, hay que indicar que el último factor -por su importancia relativa- que se engloba en este pilar es la existencia de premios y reconocimientos a las innovaciones sociales. Estos pueden ser otorgados por la administración o por entidades privadas, y, su existencia colabora a crear un ecosistema favorable al reconocer el trabajo de ciertas comunidades, aportándoles publicidad, respaldo y financiación. 


\section{Conclusiones}

El énfasis que se ha ido poniendo durante todos estos años en comprender y analizar la innovación social viene provocado por la existencia de retos sociales complejos y multidimensionales que afectan a muchos territorios. Por este motivo, se requiere un sistema de innovación de nuevo cuño que, por un lado, sea capaz de canalizar y utilizar las innovaciones tecnológicas para lograr resolver problemas sociales técnico-económicos y que, por otro lado, logre a través de la apertura y la creatividad, generar innovaciones sociales que aporten soluciones alternativas, eficientes y eficaces.

Tras el análisis realizado, entendemos que la innovación social no es un concepto utópico ni de moda, sino un fenómeno transformador que ha ido ganando fuerza hasta consolidarse. Ésta ha logrado poner de relieve una nueva forma de abordar problemas y necesidades a nivel territorial, realzando el esfuerzo de la comunidad y otorgando a la sociedad el peso que merece en la democratización económica, política y social.

Además, hemos podido comprobar que el fenómeno de la innovación social puede ser abordado desde diferentes perspectivas. Una razón de la pretendida apropiación del concepto puede vincularse al interés partidista de aquellos que desean que a sus iniciativas y prácticas se les asocien las características positivas de la innovación social transformadora. Ciertamente, ésta puede ser un complemento al sistema neoliberal, pero también una alternativa donde el colectivismo y la cooperación entre los sujetos ganan significatividad frente al individualismo y la competencia.

Nosotros la definimos como: Prácticas o iniciativas llevadas a cabo por la comunidad que, a partir de los productos, servicios o modelos que generan, o bien a través del proceso diseñado para conseguir sus objetivos, alcanzan soluciones que dan una mejor respuesta, de forma alternativa y creativa, a problemas o necesidades sociales

También es valioso destacar que la innovación social puede considerarse en distintos niveles territoriales, pero es especialmente interesante centrar el análisis en ciudades y barrios porque son los lugares donde se evidencian con mayor intensidad los problemas urbanos (pobreza, desempleo, dificultad de acceso a la vivienda, gentrificación, contaminación, entre otros) y donde la mayoría de las personas desarrollan lo fundamental de su existencia.

Así, hay que tener en cuenta que la innovación social requiere de la presencia de unas determinadas condiciones en los territorios para su desarrollo. Se reconoce de este modo, que la existencia de necesidades sociales no es el único elemento que debe acontecer para que surjan. Con nuestro trabajo hemos averiguado un conjunto de factores que contribuyen a la potenciación y al nacimiento 
de la innovación social y hemos ponderado la importancia de cada uno de ellos en función de sus frecuencias relativas de aparición. Este conocimiento está al servicio de aquellos interesados en el diseño y ejecución de políticas de innovación social y con ello se pretende facilitar la consecución de una sociedad en la que, a través de las comunidades y las iniciativas socialmente innovadoras, se logre aportar soluciones a las necesidades y retos sociales existentes.

En total hemos identificado 29 factores que se han agrupado para su presentación en cinco grandes pilares (ver tabla 2): elementos sociales y culturales; apoyo político e institucional; conocimiento y mecanismos facilitadores; componentes espaciales; y entidades y mecanismos que determinan la estructura productiva empresarial y social.

Entre los factores reconocidos, no todos pueden ser fácilmente fomentados a partir de políticas favorecedoras de la innovación social. Esto se debe a que, de todos los factores detectados, existen algunos de ellos que son estructurales, y que, en diferente grado, son necesarios que estén presentes ex-ante de cualquier política que pretenda el desarrollo de la innovación social. Entre ellos, los cinco con mayor frecuencia relativa son: que la población tenga un nivel socioeconómico medio (0.09532), que haya preocupación social (0.05719), que exista propensión cultural al cambio (0.05719), que haya propensión a la colaboración (0.03812) y que exista una clase creativa (0.03812). Por lo tanto, la puesta en marcha de cualquier acción que tenga como fin la elaboración de planes y estrategias con el fin de potenciar la innovación social, requiere de una primera fase en la que dicho tipo de factores deben ser analizados en profundidad para conocer en qué medida van a condicionar la efectividad de los planes, e incluso si pueden convertirlos en infructíferos.

Una vez realizada la primera fase del análisis, es necesaria una segunda fase basada en establecer líneas sobre aquellos factores identificados como potenciales contribuidores de la innovación social. A continuación, se señala una batería de los 10 elementos más significativos, procedentes de diferentes pilares, sobre los que sería posible diseñar una estrategia por parte de las administraciones públicas que deseen fomentar la innovación social en sus territorios: la existencia de programas de apoyo a la innovación social (0.08838), la existencia de laboratorios o centros de innovación social (0.05892), la existencia de financiación pública (0.05892), el conocimiento de la realidad local (0.05545), el conocimiento de las oportunidades y potencialidades de nuevos medios e instrumentos (0.04506), los nexos que favorecen las relaciones público-privadas (0.04332), la existencia de cooperativas (0.03986), las facilidades y medios para establecer nuevas comunicaciones y redes $(0.03812)$, los espacios para el desarrollo de las iniciativas (0.02946) y la existencia de una regulación eficiente y ausencia de trabas $(0.02946)$. Las ponderaciones de los factores pueden ser de utilidad para la priorización de las líneas en aquellas estrategias que se enfoquen en promover la innovación social.

Por último, hay que subrayar que el reconocimiento de dichos factores, junto con la ponderación realizada, supone una novedad considerable con respecto a la literatura existente. Este trabajo ha permitido comprobar la idoneidad de algunos factores determinantes que previamente ya habían sido señalados en otros estudios, enunciar otros nuevos que han emergido tras la investigación, explicar 
las relaciones entre ellos y exponer cómo de importante y qué papel juega cada uno en la generación de un ecosistema favorable para la innovación social. Este conocimiento puede resultar muy útil a la hora de establecer líneas y criterios para el diseño de estrategias de priorización, necesarias en contextos en los que existen restricciones de naturaleza política, organizacional o presupuestaria.

\section{Bibliografía}

ABREU, J.L. \& CRUZ, J.G. (2011): "Modelos de Innovación Social", Revista Daena (International Journal of Good Conscience), 6(2), 205-217.

BARRERA, E. (2007): "La empresa social y su responsabilidad social", Innovar: Revista de ciencias administrativas y sociales, 17(30), 59-76, DOI: 10.15446/innovar.v26n59.54366

BARÓN, N. \& ROMERO, J. (2018): Cultura territorial e innovación social ¿Hacia un nuevo modelo metropolitano en Europa del Sur?, Valencia.

BELDA-MIQUEL, S., BONI ARISTIZABAL, A. \& PELLICER-SIFRES, V. (2018): "Políticas públicas locales e innovación social colectiva: repensando la acción pública para acompañar la construcción de nuevos modelos sociales desde abajo". En: Barón, N. \& Romero, J., Cultura territorial e innovación social ¿Hacia un nuevo modelo metropolitano en Europa del Sur?, 47-58.

BERELSON, B. (1952): "Content Analysis in Communication Researches", Glencoe III, Free Press, DOI: $10.1177 / 000271625228300135$

BERNARDINO, S. \& SANTOS, J.F. (2017): "Local development through social and territorial innovation: An exploratory case study", CIRIEC-España, Revista de Economía Pública, Social y Cooperativa, 90, 159-187, DOI: 10.7203/CIRIEC-E.90.9155.

BLANCO, I., CRUZ, H. \& MARTINEZ, R. (2016): "El papel de la innovación social frente a la crisis", Ciudad y Territorio. Estudios Territoriales, 47(188), 249-260.

BLANCO, I. (2018): "¿Frente a la desigualdad social, innovación social? Lecciones teoricas, politicas metodologicas de una investigacion en Catalunya". En: Barón, N. \& Romero, J., Cultura territorial e innovación social ¿Hacia un nuevo modelo metropolitano en Europa del Sur?, 21-34.

BUREAU OF EUROPEAN POLICY ADVISERS (2014): Social Innovation, a decade of changes, Publications Office of the European Union 2014, Luxembourg.

CALZADA, I. (2013): "Critical social innovation in the smart city era for a city-regional european horizon 2020", P3T Journal of Public Policies \& Territories, Social Innovation and Territory, 6, 1-20. 
CARAVACA, I. (2018): "Crisis, transformaciones urbanas e innovación social en Sevilla: Contrastes y complejidades". En: En Barón, N. \& Romero, J., Cultura territorial e innovación social ¿Hacia un nuevo modelo metropolitano en Europa del Sur?, 35-46.

CAULIER-GRICE, J., DAVIES, A., PATRICK, R. \& NORMAN, W. (2012): Defining social innovation. A deliverable of the project: The theoretical, empirical and policy foundations for building social innovation in Europe (TEPSIE), European Commission-7th Framework Programme, Brussels: European Commission, DG Research.

CHESBROUGH, H.W. (2006): "Open innovation: The new imperative for creating and profiting from technology", Harvard Business Press, DOI: 10.5172/impp.2004.6.3.474.

COMISIÓN EUROPEA (2013): Guía de la Innovación social, Regional and Urban Policy, Bruselas.

DJELLAL, F. \& GALLOUJ, F. (2012): "Social innovation and service innovation. In Challenge social innovation". En: Franz, H.W., Hochgerner, J. \& Howaldt, J. (Eds), Challenge social innovation: potentials for business, social entrepreneurship, welfare and civil society, Springer Science \& Business Media, 119-137, DOI: 10.1007/978-3-642-32879-4_8.

ECHEVERRÍA, J. (2008): "El manual de Oslo y la innovación social", Arbor, 184(732), 609-618, DOI:10.3989/arbor.2008.i732.210.

ECHEVERRÍA, J. \& MERINO, L. (2011): "Cambio de paradigma en los estudios de innovación: el giro social de las políticas europeas de innovación", Arbor 187. 752: 1031-1043, DOI: 10.3989/arbor.2011.752n6002.

ECHEVERRÍA, J. \& GURRUTXAGA, A. (2012): La luz de la Luciérnaga, Plaza y Valdés, Madrid.

ESCALANTE, E. (2009): "Métodos de análisis de las verbalizaciones: una contribución del análisis textual y conceptual mediante el uso de software", Encuentro, 83, 32-48, DOI: 10.5377/encuentro.v0i83.3598.

FERRARIS, A. \& GRIECO, C. (2015): "The role of the innovation catalyst in social innovation-an Italian case study", Sinergie Italian Journal of Management, 97, 127-144.

FONTAN, J.M., KLEIN, J.L. \& LÉVESQUE, B. (2005): "The fight for jobs and economic governance: the Montreal model", Metropolitan Democracies: Transformations of the State and Urban Policy in Canada, France and Great Britain, Aldershot: Burlington, DOI: 10.4324/9781351153089-9.

FRAILE ARANDA, A., APARICIO HERGUEDAS, J.L., ASÚN DIESTE, S. \& ROMERO MARTÍND, R. (2018): "La evaluación formativa de las competencias genéricas en la formación del profesorado de educación física", Estudios pedagógicos (Valdivia), 44(2), 39-53, DOI: 10.4067/s071807052018000200039.

HARRIS, M. \& ALBURY, D. (2009): The innovation imperative. NESTA, London, DOI: 10.13007/121. 
HERNÁNDEZ-ASCANIO, J., TIRADO-VALENCIA, P. \& ARIZA-MONTES, A. (2016): "El concepto de innovación social: ámbitos, definiciones y alcances teóricos", CIRIEC-España, revista de economía pública, social y cooperativa, 88, 164-199, DOI: 10.7203/CIRIEC-E.88.8849.

HILLIER, J., MOULAERT, F. \& NUSSBAUMER, J. (2004): "Trois essais sur le rôle de l'innovation sociale dans le développement territorial", Géographie, économie, société, 6(2), 129-152. DOI: 10.3166/ges.6.129-152.

HOWALDT, J. \& SCHWARZ, M. (2010): Social Innovation: Concepts, research fields and international trends, Sozialforschungsstelle Dortmund.

HOWALDT, J., SCHRÖDER, A., KALETKA, C., REHFELD, D. \& TERSTRIEP, J. (2016): Mapping the world of social innovation, A deliverable key result of a comparative analysis of the project SIDRIVE. European Commission-8th Framework Programme, Brussels: European Commission, DG Research.

HUBERT, A. (2010): Empowering people, driving change: Social innovation in the European Union, Bureau of European Policy Advisors.

KRLEV, G., BUND, E. \& MILDENBERGER, G. (2014): "Measuring what matters-Indicators of social innovativeness on the national level". Information Systems Management, 31(3), 200-224, DOI: $10.1080 / 10580530.2014 .923265$.

LÓPEZ, J.A. \& GONZALEZ, M. (2013): "Encrucijadas sociales de la innovación”, Isegoría, 48, 11-24, DOI: 10.3989/isegoria.2013.048.01.

LUNDVALL, B.A. (1988): "Innovation as an interactive process: from user-producer interaction to national systems of innovation", Technical change and economic theory.

MACCALLUM, D. (Ed.) (2009): Social innovation and territorial development. Ashgate Publishing, Ltd., DOI: 10.4324/9781315609478.

MÉNDEZ, R. (2002): Innovación y desarrollo territorial: algunos debates teóricos recientes. Eure (Santiago), 28(84), 63-83, DOI: 10.4067/s0250-71612002008400004.

MOSSELAER, F. (2016): Creative Industries: Social Innovation. Academy for creative industries, DOI:10.4337/9780857930705.00014.

MOULAERT, F., MARTINELLI, F. \& SWYNGEDOUW (2004): Social innovation and governance in local communities, SINGOCOM Final report to the EC (FP6), Lille: IFRESI.

MOULAERT, F., MARTINELLI, F., SWYNGEDOUW, E. \& GONZALEZ, S. (2005): "Towards alternative model (s) of local innovation", Urban studies, 42(11), 1969-199, DOI: 10.1080/00420980500279893.

MOULAERT, F., MARTINELLI, F., GONZÁLEZ, S. \& SWYNGEDOUW, E. (2007): "Introduction: social innovation and governance in European cities urban development between path dependency and radical innovation", European Urban and Regional Studies, 14(3), 195-209, DOI: 10.1177/0969776407077737. 
MOULAERT, F., MACCALLUM, D. \& HILLIER, J. (2013): Social innovation: intuition, precept, concept. The International Handbook on Social Innovation: collective action, social learning and transdisciplinary research, 13, DOI: 10.4337/9781849809986.00011.

MULGAN, G. (2006): "The process of social innovation”, Innovations: technology, governance, globalization, 1(2), 145-162, DOI: 10.1162/itgg.2006.1.2.145.

NAVARRO, C. (2015): "Innovación social y gobernanza urbana". En: Subirats, J. \& García Bernardos, A. Eds. (2015), 43-58.

NELSON, R. \& ROSENBERG, N. (1993): "Technical innovation and national systems. National innovation systems: a comparative analysis", Oxford University Press, 7, 1-18.

NESTA (2008): Social innovation: New approaches to transforming public services. Making Innovation Flouurish, London.

NEUMEIER, S. (2017): "Social innovation in rural development: identifying the key factors of success". The geographical journal, 183(1), 34-46, DOI: 10.1111/geoj.12180.

NOGUERO, F. (2002): "El análisis de contenido como método de investigación", Revista de Educación, 4, 167-179, DOI: 10.23878/empr.v12i45.129.

OCDE (2005): Oslo Manual: Guidelines for Collecting and Interpreting Innovation Data, 3d, Committee for Scientific and Technological Policy, OCDE, Paris, DOI: 10.1787/9785760201737-ru.

OCDE (2011): Fostering Innovation to Address Social Challenges. Workshop proceedings. OECD Innovation Strategy, DOI: 10.1016/j.techfore.2015.08.011.

OOSTERLYNCK, S., KAZEPOV, Y., NOVY, A., COOLS, P., BARBERIS, E., WUKOVITSCH, F. \& LEUBOLT, B. (2013): "The butterfly and the elephant: local social innovation, the welfare state and new poverty dynamics", ImPRovE Methodological Paper, (13/02).

PALMA, L., GARCÍA-FLORES, V. \& FERNÁNDEZ, I. (2017): "La Agencia de Defensa de la Competencia de Andalucía como agente de innovación social en el marco de la Estrategia Europea de Especialización Inteligente", Comunicación presentada en el XVIII Congreso de Economía Regional celebrado en Sevilla, los días 15,16 y 17 de noviembre de 2017.

PAZ, E. (2015): "Un paradigma emergente: la innovación social”, 3C Empresa, 4 (1), 50-68.

PÉREZ-LÓPEZ, R., MORALES-SANCHEZ, M., ANGUERA, T. \& HERNÁNDEZ-MENDO, A. (2015): "Evaluación de la calidad total en servicios municipales deportivos orientados a la población infantil: Aportaciones desde el análisis cualitativo con Atlas.ti", Cuadernos de Psicología del Deporte, 15(1), 143-150, DOI: 10.4321/s1578-84232015000100014.

PHILLS, J.A., DEIGLMEIER, K. \& MILLER, D.T. (2008): "Rediscovering social innovation”, Stanford Social Innovation Review, 6(4), 34-43.

POT, F. \& VAAS, F. (2008): "Social innovation, the new challenge for Europe", International Journal of Productivity and Performance Management, 57(6), 468-473, DOI: 10.1108/17410400810893400. 
PRADOS, J.A. (2007): "Críticas y apoyos hacia la responsabilidad social de la empresa: una aproximación etnográfica desde la vivencia del trabajador y una propuesta metodológica cualitativa". Universitas psychologica, 6(1), 59-68.

RÜEDE, D. \& LURTZ, K. (2012): "Mapping the various meanings of social innovation: Towards a differentiated understanding of an emerging concept", EBS Business School Research Paper, 1203, DOI: 10.2139/ssrn.2091039.

SALOM-CARRASCO, J., PITARCH-GARRIDO, M.D. \& SALES-TEN, A. (2017): "Innovación social: estrategias urbanas en un contexto de cambio. El caso de la ciudad de Valencia", CIRIEC-España, Revista de Economía Pública, Social y Cooperativa, 91, 31-58, DOI: 10.7203/CIRIEC-E.91.10451.

SANCHÍS, J.R. \& CAMPOS, V. (2008): "La innovación social en la empresa. El caso de las cooperativas y de las empresas de Economía Social en España”, Economía Industrial, 368, 187-196.

SUBIRATS, J. \& GARCÍA BERNARDOS, A. (Eds.) (2015): Innovación social y políticas urbanas en España: experiencias significativas en las grandes ciudades, ICARIA, Barcelona.

SUBIRATS, J. (2015): "Políticas urbanas e innovación social. Entre la coproducción y la nueva institucionalidad. Criterios de significatividad". En: Subirats, J. \& García Bernardos, A., Eds., Innovación social y políticas urbanas en España: experiencias significativas en las grandes ciudades, 13-42.

UNCETA, A., CASTRO-SPILA, J. \& GARCÍA, J. (2016): "Social innovation indicators". Innovation: The European Journal of Social Science Research, 29(2), 192-204, DOI: 10.1080/13511610.2015.1127137.

VAN DYCK, B. \& VAN DEN BROECK, P. (2013): "Social innovation: a territorial process", The international handbook on social innovation: collective action, social learning and transdisciplinary research, 131-141. Edward Elgar, Cheltenham, UK. DOI: 10.4337/9781849809993.00021.

VRONTIS, D., THRASSOU, A., CHEBBI, H. \& YAHIAOUI, D. (2012): "Transcending innovativeness towards strategic reflexivity", Qualitative Market Research: An International Journal, 15(4), 420437, DOI: 10.1108/13522751211257097,

WORLD ECONOMIC FORUM \& SCHWAB FOUNDATION FOR SOCIAL ENTREPRENEURSHIP (2016): Social Innovation: A Guide to Achieving Corporate and Societal Value, Cologny/Geneva Switzerland.

YEPEZ, F. (2017): La Estrategia Europea de Especialización Inteligente. Implicaciones para el Sistema Andaluz de Innovación, Tesis Doctoral, Universidad de Sevilla.

ZUBERO, I. (2015): "Innovación social: una propuesta para pensar las prácticas sociales en clave de transformación". En: Subirats, J. \& García Bernardos, A., Eds., Innovación social y políticas urbanas en España: experiencias significativas en las grandes ciudades, 13-42. 


\section{Anexo 1: Personas que han sido entrevistadas para la realización del trabajo}

\begin{tabular}{|c|c|}
\hline $\begin{array}{l}\text { Responsables de centros } \\
\text { o empresas }\end{array}$ & Posición \\
\hline Reyes García & Agente de innovación local de la red Guadalinfo. \\
\hline Ana María Romero & Directora del Centro de Innovación Social de la provincia de Málaga. \\
\hline Javier González & Director del laboratorio de innovación social y digital "Citilab Cornellà" \\
\hline Carlos Lora & Cofundador y presidente del laboratorio de innovación social "Espacio_Res" \\
\hline David Pino & Director de Innovación y Economía Social en el Ayuntamiento de Sevilla \\
\hline Gotzon Bernaola & Director de Programas de Innovación Social en Innobasque (Agencia Vasca de Innovación) \\
\hline Casilda Heraso & Directora de Selección de Emprendedores en Ashoka \\
\hline Ona Argemí & Es miembro del equipo de la consultora UpSocial con sede operativa en Barcelona. \\
\hline Resurrección Hernández & Directora del Área de Servicios a la Ciudadanía de la Diputación Provincial de Málaga \\
\hline Académicos & Posición \\
\hline Jenny Melo & Investigadora y coautora del trabajo "Panorama actual de la Innovación Social en Colombia". \\
\hline Ismael Blanco & $\begin{array}{l}\text { Profesor asociado del Departamento de Ciencia Política e investigador del Instituto de Gobierno } \\
\text { y Políticas Públicas de la Universidad Autónoma de Barcelona. }\end{array}$ \\
\hline Ana Luisa López & Investigadora en el Departamento de Didáctica y Organización Escolar en la Univ. de Deusto. \\
\hline Laura Villa & Investigadora y coautora del trabajo "Panorama actual de la Innovación Social en Colombia". \\
\hline Carlos María & Investigador en el Departamento de Economía Aplicada de la Universidad de Vigo. \\
\hline Pilar Tirado & $\begin{array}{l}\text { Investigadora en el departamento de Economía Financiera y Contabilidad. Miembro del grupo } \\
\text { de investigación en economía e innovación social. -SOCIAL MATTERS }\end{array}$ \\
\hline Directores y encargados & Posición \\
\hline Ana Hevilla Ordóñez & Responsable del Área de Desarrollo Rural del Grupo del Valle del Guadalhorce. \\
\hline Luis Miguel Jurado & Presidente de la Federación Andaluza de Empresas Cooperativas de Trabajo (FAECTA) \\
\hline Jose Manuel Salado & Persona encargada de la iniciativa de Innovación Social "Crafteando". \\
\hline Javier Montaner & Director de Mouse4all. \\
\hline Juan Antonio Vallecillo & Asistente de proyectos en Asprodisis. \\
\hline Elena Domínguez & Directora en España de la iniciativa Perros y Letras en España. \\
\hline Rubén Martínez & Coordinador del área de investigación de la Hidra Cooperativa. \\
\hline Oriol Vilalta i Caellas & Director general de la Fundación "Pau Costa". \\
\hline Maria Vela & Coordinadora del área de Agroecología y Permacultura en Ecoherencia. \\
\hline
\end{tabular}




\section{Anexo 2: Guión utilizado para la realización de las entrevistas semiestructuradas a los diferentes agentes económicos y sociales}

\section{PARTE I: Preguntas genéricas sobre innovación social}

Desde su punto de vista, ¿qué es para usted la innovación social?

¿Cuáles cree que son las principales características y/o propiedades que debe tener una iniciativa 0 actividad para considerarla como una innovación social?

¿Cree que se está sobre utilizando el concepto de innovación social? ¿Teme que pierda su valor?

¿Qué motivos cree que llevan a los agentes a realizar prácticas socialmente innovadoras? En su caso o el de su asociación/empresa/sector representado, ¿cuál fue su motivación principal?

¿Qué papel cree que juegan en la innovación social los centros de apoyo a la innovación social? ¿Y los académicos? ¿Señalaría algún otro agente que fuera relevante para el fomento y desarrollo de las innovaciones sociales? ¿Qué tipo de apoyo ha recibido su asociación /empresa?

¿Cuál cree que es el perfil de los agentes socialmente innovadores? ¿Qué tipo de organizaciones, empresas (centros tecnológicos, empresariales, cooperativas...), organismos públicos cree que pueden llevar a cabo actividades socialmente innovadoras?

¿Qué etapas cree que suele pasar una iniciativa socialmente innovadora desde que nace hasta que se consolida? ¿Cuál es la fase más crítica?

\section{PARTE II: Identificación de variables}

¿En qué tipo de regiones cree que se desarrollan las innovaciones sociales?

¿Cree que en aquellas regiones donde existen más problemas, surgen más innovaciones sociales?

¿Qué factores facilitaron el lanzamiento de su iniciativa socialmente innovadora?

¿Qué factores supusieron una barrera o dificultaron dicho proceso?

Si tuviese que elegir tres grandes pilares que son esenciales para el desarrollo y fomento de las innovaciones sociales, ¿cuáles serían?

¿Cómo cree que pueden ser medidos dichos factores?

¿Quién cree que puede promover que esos factores que usted menciona puedan ser incentivados?

¿Qué papel considera que tiene la creatividad sobre la innovación social? ¿Y el arte y la cultura?

\section{PARTE III: Las potencialidades de la innovación social}

¿Cuáles cree que son los principales beneficios de llevar a cabo actividades socialmente innovadoras para la sociedad?

¿En qué campos cree que puede ser especialmente útil o relevante el desarrollo de innovaciones sociales? ¿Qué tipo de problemas cree que puede coadyuvar a resolver?

¿Cree que puede ser afirmado que aquellas regiones donde existen menos problemas es porque hay iniciativas socialmente innovadoras? ¿O cree que la poca incidencia de determinados problemas en una región precisamente desincentiva la innovación social?

¿Qué papel cree que tiene la innovación social en la mejora y la modernización del sector público?

¿Qué implicaciones positivas cree que su iniciativa genera en la sociedad?

¿Cuál es el objetivo de su iniciativa/centro/grupo de investigación a medio plazo? 